\title{
SETS OF INDEPENDENT POSTULATES FOR THE ARITHMETIC MEAN, THE GEOMETRIC MEAN, THE HARMONIC MEAN, AND THE ROOT-MEAN-SQUARE*
}

BY

EDWARD V. HUNTINGTON

\section{INTRODUCTION}

The four types of means, or averages, considered in this paper are the following: the arithmetic mean $(A)$; the geometric mean $(G)$; the harmonic mean $(H)$; and the root-mean-square $(S)$; the familiar definitions being as follows:

$$
\begin{aligned}
A= & \frac{1}{n}\left(x_{1}+x_{2}+\cdots+x_{n}\right), \\
G= & \left(x_{1} x_{2} \cdots x_{n}\right)^{1 / n} \\
H= & \frac{1}{\frac{1}{n}\left(\frac{1}{x_{1}}+\frac{1}{x_{2}}+\cdots+\frac{1}{x_{n}}\right)}, \\
S= & \left(\frac{1}{n}\left(x_{1}^{2}+x_{2}^{2}+\cdots+x_{n}^{2}\right)\right)^{1 / 2} .
\end{aligned}
$$

The first three are the classical means, known to the Greeks, while the rootmean-square is of more modern origin. Of the four types, perhaps the most important are the arithmetic mean and the root-mean-square. Both of these averages are in constant use in mechanics (as in the definitions of center of gravity and radius of gyration), and in the modern theory of statistics (as in the theory of probability, the theory of least squares, and the definition of standard deviation). The geometric mean is important chiefly in the construction of index numbers. The harmonic mean is little used, except in special investigations.

Each of the four quantities $A, G, H, S$ is a particular type of the general function of $n$ variables: $f\left(x_{1}, x_{2}, \cdots, x_{n}\right)$; and the purpose of the present

*Presented to the Society, December 29, 1925; received by the editors April 5, 1926.

EDITOR'S NOTE. The typography in this paper and succeeding papers has been altered in various respects from the form originally proposed by the authors, in an effort to adapt mathematical composition to the monotype machine. 
paper is to exhibit a number of sets of independent postulates by which each of these four types may be distinguished.

Unless otherwise stated, the variables $x_{1}, x_{2}, \cdots, x_{n}$ are supposed to be positive real numbers.

The only similar set of postulates for any of these means, as far as is known to the present writer, is a set of postulates for the arithmetic mean given by R. Schimmack* in 1909; the "complete independence" of Schimmack's postulates was established by R. D. Beetle $\dagger$ in 1915.

\section{Properties COMMON TO ALL FOUR MEANS}

It will be convenient to begin by stating the following general postulates, $\mathrm{I}-\mathrm{V}$, which are satisfied by all four of the types of mean here considered. Each of these postulates is a condition imposed upon the as yet undetermined function $f\left(x_{1}, x_{2}, \cdots, x_{n}\right)$ of the $n$ positive real numbers $x_{1}, x_{2}, \cdots, x_{n}$. Various selections from these postulates will be made below.

I. $f\left(x_{1}, x_{2}, \cdots, x_{i}, x_{i}, \cdots, x_{n}\right)=f\left(x_{1}, x_{2}, \cdots, x_{i}, x_{i}, \cdots, x_{n}\right)$.

That is, the function " $f$ " is independent of the order in which the $n$ quantities $x_{1}, x_{2}, \cdots, x_{n}$ are taken.

II. $f\left(x_{1}, x_{2}, x_{3}, \cdots, x_{n}\right)=f\left(m, m, x_{3}, \cdots, x_{n}\right)$ where $m=f\left(x_{1}, x_{2}\right)$.

That is, in computing the " $f$ " of $n$ quantities, we may replace the first pair, $x_{1}, x_{2}$, by the " $f$ " of that pair, entered twice.

III. $f\left(k x_{1}, k x_{2}, \cdots, k x_{n}\right)=k f\left(x_{1}, x_{2}, \cdots, x_{n}\right) \quad$ ( $k$ positive $)$.

That is, multiplying each of the $n$ quantities by a positive factor $k$ has the effect of multiplying the " $f$ " of those quantities by the same factor $k$. In other words, the function " $f$ " is independent of the scale in which the quantities $x_{1}, x_{2}, \cdots, x_{n}$ are measured.

IV.

$$
f(a, a, \cdots, a)=a .
$$

That is, if the $n$ quantities are all equal, then their " $f$ " is equal to their common value.

V. $f\left(x_{1}, x_{2}, \cdots, x_{n}\right)$ is positive when all the $x$ 's are positive.

The postulates III and IV may sometimes be replaced, as we shall see, by the following weaker forms:

*R. Schimmack, Der Satz vom arithmetischen Mittel in axiomatischer Begründung, Mathematische Annalen, vol. 68 (1909), pp. 125-132, and p. 304.

$\dagger \mathrm{R}$. D. Beetle, On the complete independence of Schimmack's postulates for the arithmetic mean, Mathematische Annalen, vol. 76 (1915), pp. 444-446. 
III'. $\quad f\left(k x_{1}, k x_{2}\right)=k f\left(x_{1}, x_{2}\right) \quad(k$ positive $)$.

IV'. $\quad f(1,1, \cdots, 1)=1$.

It may be noted that Postulates I, II, III, and III' have the form of "functional equations," in which the function " $f$ " appears on both sides of the equality sign; while Postulates IV, V, and IV' are analogous to the "boundary conditions" of a problem in differential equations, since they tell us something about the actual value of the function in certain cases.

We now turn to properties which are peculiar to the several types of mean. (It will be observed that in each of the following four groups, the first postulate is a "boundary condition," while the other three postulates are "functional equations.") Various selections from these postulates will be made below.

Postulates PeCUliar to THE ARIthmetic MeAN (POSITIVe QUANTITIEs*) $A 1$.

$$
f(a, b)=\frac{1}{2}(a+b) \text {. }
$$

$A 2$.

$$
f(1-a, 1-b)=1-f(a, b) \quad(a<1, b<1) \text {. }
$$

A3. $f\left(1-x_{1}, 1-x_{2}, \cdots, 1-x_{n}\right)=1-f\left(x_{1}, x_{2}, \cdots, x_{n}\right)\left(x_{i}<1\right)$.

A4. $f\left(A-x_{1}, A-x_{2}, \cdots, A-x_{n}\right)=A-f\left(x_{1}, x_{2}, \cdots, x_{n}\right)$ for all values of $A$ for which $A-x_{i}>0$.

Postulates Peculiar to the geometric MEAN

G1.

$$
f(a, b)=(a b)^{1 / 2} \text {. }
$$

G2.

$$
f\left(\frac{1}{a}, \frac{1}{b}\right)=\frac{1}{f(a, b)} \text {. }
$$

G3.

$$
f\left(\frac{1}{x_{1}}, \frac{1}{x_{2}}, \ldots, \frac{1}{x_{n}}\right)=\frac{1}{f\left(x_{1}, x_{2}, \cdots, x_{n}\right)} \text {. }
$$

G4.

$$
f\left(\frac{A}{x_{1}}, \frac{A}{x_{2}}, \ldots, \frac{A}{x_{n}}\right)=\frac{A}{f\left(x_{1}, x_{2}, \cdots, x_{n}\right)},
$$

where $A$ is positive. Here $(a b)^{1 / 2}$ means $+(a b)^{1 / 2}$ not $-(a b)^{1 / 2}$.

\section{Postulates peculiar to THE haRmonic MeaN}

H1.

$$
f(a, b)=\frac{2 a b}{a+b}
$$

'For further postulates $A 8, A 8$ ', $A 9$, intended for use in the domain of all real or all complex quantities, see Appendix I and Appendix II. 
H2.

$$
\left(\frac{a}{a-1}, \frac{b}{b-1}\right)=\frac{f(a, b)}{f(a, b)-1} .
$$

H3. $f\left(\frac{x_{1}}{x_{1}-1}, \frac{x_{2}}{x_{2}-1}, \ldots, \frac{x_{n}}{x_{n}-1}\right)=\frac{f\left(x_{1}, x_{2}, \cdots, x_{n}\right)}{f\left(x_{1}, x_{2}, \cdots, x_{n}\right)-1}\left(x_{i}>1\right)$.

H4. $f\left(\frac{x_{1}}{A x_{1}-1}, \frac{x_{2}}{A x_{2}-1}, \ldots, \frac{x_{n}}{A x_{n}-1}\right)=\frac{f\left(x_{1}, x_{2}, \cdots, x_{n}\right)}{A f\left(x_{1}, x_{2}, \cdots, x_{n}\right)-1}$

for all values of $A$ for which $A x_{i}-1>0$.

Postulates PECULIAR TO THE ROOT-MEAN-SQUARE

$S 1$.

$$
f(a, b)=\left\{\left(x_{1}^{2}+x_{2}^{2}\right) / 2\right\}^{1 / 2} .
$$

S2.

$$
f\left\{\left(1-a^{2}\right)^{1 / 2},\left(1-b^{2}\right)^{1 / 2}\right\}=\left\{1-[f(a, b)]^{2}\right\}^{1 / 2} \quad(a<1, b<1) .
$$

S3. $f\left\{\left(1-x_{1}^{2}\right)^{1 / 2},\left(1-x_{2}^{2}\right)^{1 / 2}, \cdots,\left(1-x_{n}^{2}\right)^{1 / 2}\right\}$

$$
=\left\{1-\left[f\left(x_{1}, x_{2}, \cdots, x_{n}\right)\right]^{2}\right\}^{1 / 2}\left(x_{i}<1\right) .
$$

S4. $\quad f\left\{f\left(A-x_{1}^{2}\right)^{1 / 2},\left(A-x_{2}^{2}\right)^{1 / 2}, \cdots,\left(A-x_{n}^{2}\right)^{1 / 2}\right\}$

$$
=\left\{A-\left[f\left(x_{1}, x_{2}, \cdots, x_{n}\right)\right]^{2}\right\}^{1 / 2},
$$

for all values of $A$ for which $A-x_{i}{ }^{2}>0$. Here again, $x^{1 / 2}$ means $+x^{1 / 2}$ not $-x^{1 / 2}$.

\section{SETS OF INDEPENDENT POSTULATES FOR EACH TYPE OF MEAN.}

Among these general and special properties there are, of course, many redundancies. The purpose of the present paper is to select, for each type of mean, sets of independent postulates; that is, sets of postulates which, while sufficient to determine uniquely the type of mean in question, shall at the same time be free from all redundancies. Such sets can be selected in a variety of ways, as in the following tables. These tables give the postulates belonging to each set, and also the list of examples which will later be used to prove the independence of the postulates in that set.

It will be observed that in each group the fifth and sixth sets are obtained from the second set by replacing either III or IV by the weaker form III' or IV'; while the seventh set is obtained from the first by replacing IV by IV' and then adding III. No further replacements of III or IV by III' or IV' are possible, as we shall see by Examples $A$ III 7, $G$ III 7, $H$ III 7, and $S$ III 7.

Thus, a set of postulates comprising $A 1, A 2, A 3, \mathrm{I}, \mathrm{II}, \mathrm{III}^{\prime}, \mathrm{IV}^{\prime}, \mathrm{V}$ would not be sufficient to determine the arithmetic mean; G1, G2, G3, I, II, III',

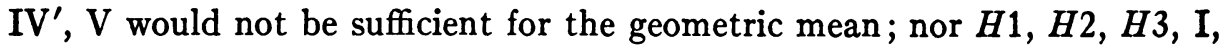
II, III', IV', V for the harmonic mean; nor $S 1, S 2, S 3, \mathrm{I}, \mathrm{II}, \mathrm{III}^{\prime}, \mathrm{IV}^{\prime}, \mathrm{V}$ for the root-mean-square. 
For the Arithmetic Mean (positive quantities*)

\begin{tabular}{|c|c|c|c|c|c|c|c|c|c|c|}
\hline Set & \multicolumn{5}{|c|}{ Postulates } & \multicolumn{5}{|c|}{ Examples Used } \\
\hline$A 1$ & $A 1$ & I & II & & IV & 0 & $A \mathrm{I}$ & $A$ II & & $A$ IV \\
\hline$A 2$ & $A 2$ & I & II & III & IV & 0 & $A \mathrm{I}$ & $A$ II & $A$ III & $A \mathrm{IV}$ \\
\hline$A 3$ & $A 3$ & I & II & III & & 0 & $A \mathrm{I}$ & $A$ II & $A$ III & \\
\hline$A 4$ & $A 4$ & I & II & & & 0 & $A \mathrm{I}$ & $A$ II & & \\
\hline$A 5$ & $A 2$ & I & II & III' $^{\prime}$ & IV & $\mathbf{0}$ & $A \mathrm{I}$ & $A$ II & $A$ III & $A \mathrm{IV}$ \\
\hline$A 6$ & $A 2$ & I & II & III & IV $^{\prime}$ & 0 & $A \mathrm{I}$ & $A \mathrm{II}$ & $A \mathrm{III}$ & $A \mathrm{IV}$ \\
\hline$A 7$ & $A 1$ & I & II & III & IV' & 0 & $A \mathrm{I}$ & $A$ II & $A$ III & $A \mathrm{IV}$ \\
\hline
\end{tabular}

For the Geometric Mean

\begin{tabular}{|c|c|c|c|c|c|c|c|c|c|c|c|c|}
\hline Set & \multicolumn{6}{|c|}{ Postulates } & \multicolumn{6}{|c|}{ Examples Used } \\
\hline$G 1$ & $G 1$ & I & II & & IV & & 0 & GI & $G$ II & & $G$ IV & \\
\hline$G 2$ & G2 & I & II & III & IV & V & 0 & GI & $G \mathrm{II}$ & $G$ III & $G \mathrm{IV}$ & $G \mathrm{~V}$ \\
\hline G3 & G3 & I & II & III & & V & 0 & $G I$ & $G$ II & $G$ III & & $G \mathrm{~V}$ \\
\hline$G 4$ & G4 & I & II & & & V & 0 & GI & $G$ II & & $G \mathrm{IV}$ & $G \mathrm{~V}$ \\
\hline G5 & $G 2$ & I & II & III' $^{\prime}$ & IV & V & 0 & $G I$ & $G$ II & $G$ III & $G$ IV & $\boldsymbol{G} \mathrm{V}$ \\
\hline G6 & G2 & I & II & III & IV $^{\prime}$ & v & 0 & GI & $G$ II & $G$ III & $G$ IV & $G \mathrm{~V}$ \\
\hline G7 & $G 1$ & I & II & III & IV' & & 0 & GI & $G$ II & $G$ III & $G \mathrm{IV}$ & \\
\hline
\end{tabular}

For the Harmonic Mean

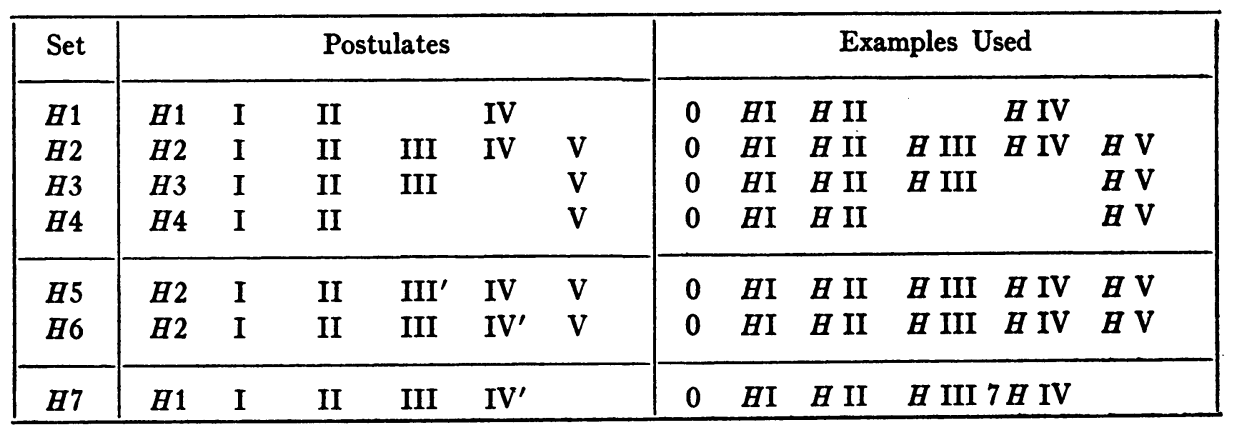

*For further Sets $A 6^{\prime \prime}, A 7^{\prime \prime}, A 8, A 9$, intended for use in the real or complex domain, see Appendices I and II. 
For the Root-Mean-Square

\begin{tabular}{|c|c|c|c|c|c|c|c|c|c|c|}
\hline Set & \multicolumn{5}{|c|}{ Postulates } & \multicolumn{5}{|c|}{ Examples Used } \\
\hline$S 1$ & $S 1$ & I & II & & IV & 0 & $S I$ & $S$ II & & $S$ IV \\
\hline$S 2$ & $S 2$ & I & II & III & IV & 0 & $S I$ & $S$ II & $S$ III & $S$ IV \\
\hline$S 3$ & $S 3$ & I & II & III & & 0 & $S I$ & $S$ II & $S$ III & \\
\hline$S 4$ & $S 4$ & I & II & & & 0 & $S I$ & $S$ II & & \\
\hline$S 5$ & $S 2$ & I & II & III' & IV & 0 & $S I$ & $S$ II & $S$ III & $S$ IV \\
\hline$S 6$ & $S 2$ & I & II & III & IV $^{\prime}$ & 0 & $S I$ & $S$ II & $S$ III & $S \mathrm{IV}$ \\
\hline$S 7$ & $S 1$ & I & II & III & IV' & $\mathbf{0}$ & $S \mathrm{I}$ & $S$ II & $S \mathrm{III} 7$ & $S$ IV \\
\hline
\end{tabular}

EXAMPLES USED IN PROOFS OF INDEPENDENCE

To establish the independence of the postulates of each set, we exhibit, in the usual way, a list of examples of functions $f\left(x_{1}, x_{2}, \cdots, x_{n}\right)$ which satisfy some but not all of the postulates. In the following table the postulates satisfied by each example are stated explicitly, opposite the number of that example. (A dash, - indicates that the postulate is not satisfied.) The list of examples is given immediately below the table.*

As an illustration of the use of these examples, consider Postulate III in Set G3. Example $G$ III satisfies Postulates G3, I, II, and V, but fails on Postulate III. Hence III is not a consequence of G3, I, II, V; that is, Postulate III is not a redundancy in Set G3. Similarly for each of the other postulates in this set and in each of the other sets.

Ex. 0.

$$
f()=\left\{\left(x_{1}^{3}+x_{2}^{3}+\cdots+x_{n}^{3}\right) / n\right\}^{1 / 3} .
$$

This example satisfies all the general postulates I-V, but none of the special postulates $A 1-A 4, G 1-G 4, H 1-H 4, S 1-S 4$. To see that II is satisfied, note that

$$
m=f\left(x_{1}, x_{2}\right)=\left\{\left(x_{1}^{3}+x_{2}^{3}\right) / 2\right\}^{1 / 3},
$$

so that $m^{3}+m^{3}=x_{1}{ }^{3}+x_{2}{ }^{3}$.

$$
\text { Ex. } A \mathrm{I} . \quad f()=\frac{x_{1}+x_{2}+3 x_{8}+4 x_{4}+\cdots+n x_{n}}{1+1+3+4+\cdots+n} .
$$

"For further Examples: $0^{\prime}, A \mathrm{II}^{\prime}, A \mathrm{III}^{\prime \prime}, A \mathrm{IV}^{\prime}, A \mathrm{IV}^{\prime \prime} ; A \mathrm{II}^{\prime \prime}, A \mathrm{IX}$, and $A \mathrm{X}$, for the arith metic mean in the real or complex domain, see Appendices I and II. 


\begin{tabular}{|l|cccc|cccc|cc|c|}
\hline & $A 1$ & $A 2$ & $A 3$ & $A 4$ & I & II & III & IV & III' $^{\prime}$ & IV' $^{\prime}$ & V \\
\hline Ex. 0 & - & - & - & - & I & II & III & IV & III' $^{\prime}$ & IV' $^{\prime}$ & V \\
Ex. $A$ I & $A 1$ & $A 2$ & $A 3$ & $A 4$ & - & II & III & IV & III' $^{\prime}$ & IV' $^{\prime}$ & V \\
Ex. $A$ II & $A 1$ & $A 2$ & $A 3$ & $A 4$ & I & - & III & IV & III' $^{\text {IV }^{\prime}}$ & V \\
\hline Ex. $A$ III & - & $A 2$ & $A 3$ & - & I & II & - & IV & - & IV' $^{\prime}$ & V \\
Ex. $A$ IV & $A 1$ & $A 2$ & - & - & I & II & III & - & III' $^{\prime}$ & - & V \\
Ex. $A$ III 7 & $A 1$ & $A 2$ & $A 3$ & - & I & II & - & - & III' $^{\prime}$ & IV' $^{\prime}$ & V \\
\hline
\end{tabular}

\begin{tabular}{|c|c|c|c|c|c|c|c|c|c|c|c|}
\hline & $G 1$ & $G 2$ & G3 & $G 4$ & I & II & III & IV & III' & IV' & V \\
\hline Ex. 0 & - & - & - & - & I & II & III & IV & III' & $\mathrm{IV}^{\prime}$ & V \\
\hline Ex. G I & $G 1$ & $G 2$ & G3 & G4 & - & II & III & IV & III' & IV' & V \\
\hline Ex. $G$ II & $G 1$ & G2 & G3 & $G 4$ & I & - & III & IV & III' & IV $^{\prime}$ & V \\
\hline Ex. $G$ III & - & $G 2$ & G3 & - & I & II & - & IV & - & IV $^{\prime}$ & V \\
\hline Ex. $G$ IV & $G 1$ & $G 2$ & - & - & I & II & III & - & $\mathrm{III}^{\prime}$ & - & V \\
\hline Ex. $G$ III 7 & $G 1$ & $G 2$ & G3 & - & I & II & - & - & $\mathrm{III}^{\prime}$ & IV $^{\prime}$ & V \\
\hline Ex. $G \mathrm{~V}$ & - & $G 2$ & G3 & G4 & I & II & III & IV & III' $^{\prime}$ & IV' & - \\
\hline
\end{tabular}

\begin{tabular}{|c|c|c|c|c|c|c|c|c|c|c|c|}
\hline & $H 1$ & $H 2$ & H3 & $H 4$ & I & II & III & IV & III' & $\mathrm{IV}^{\prime}$ & V \\
\hline Ex. 0 & - & - & - & - & I & II & III & IV & III' $^{\prime}$ & IV $^{\prime}$ & V \\
\hline Ex. $B$ I & $B 1$ & $H 2$ & $H 3$ & $H 4$ & - & II & III & IV & III' & IV' & V \\
\hline Ex. $\boldsymbol{B}$ II & $H 1$ & $H 2$ & $H 3$ & ' $H 4$ & I & - & III & IV & III' $^{\prime}$ & IV $^{\prime}$ & V \\
\hline Ex. $B$ III & - & $H 2$ & $B 3$ & - & I & II & - & IV & - & IV' & $\mathrm{v}$ \\
\hline Ex. $H$ IV & $H 1$ & H2 & - & - & I & II & III & - & III' & - & V \\
\hline Ex. $B$ III 7 & $H 1$ & $H 2$ & $\boldsymbol{H 3}$ & - & I & II & - & - & III' $^{\prime}$ & $\mathrm{IV}^{\prime}$ & V \\
\hline Ex. $H \mathrm{~V}$ & - & $H 2$ & $H 3$ & $B 4$ & I & II & III & IV & $\mathrm{III}^{\prime}$ & IV $^{\prime}$ & - \\
\hline
\end{tabular}

\begin{tabular}{|l|cccc|cccc|cc|c|}
\hline & $S 1$ & $S 2$ & $S 3$ & $S 4$ & I & II & III & IV & III' $^{\prime}$ & IV' $^{\prime}$ & V \\
\hline Ex. 0 & - & - & - & - & I & II & III & IV & III' $^{\prime}$ & IV' $^{\prime}$ & V \\
Ex. $S$ I & $S 1$ & $S 2$ & $S 3$ & $S 4$ & - & II & III & IV & III' $^{\prime}$ & IV' $^{\prime}$ & V \\
Ex. $S$ II & $S 1$ & $S 2$ & $S 3$ & $S 4$ & I & - & III & IV & III' $^{\prime}$ & IV' $^{\prime}$ & V \\
\hline Ex. $S$ III & - & $S 2$ & $S 3$ & - & I & II & - & IV & - & IV' & V \\
Ex. $S$ IV & $S 1$ & $S 2$ & - & - & I & II & III & - & III' $^{\prime}$ & - & V \\
Ex. $S$ III 7 & $S 1$ & $S 2$ & $S 3$ & - & I & II & - & - & III' $^{\prime}$ & IV' $^{\prime}$ & V \\
\hline
\end{tabular}


Ex. $A$ II. $f()=$ the "median" of the quantities $x_{1}, x_{2}, \cdots, x_{n}$, if $n$ is odd, or the arithmetic mean of the "median-pair" if $n$ is even.* To see that II fails note that $f(5,3,7)=5$, while $f(4,4,7)=4$.

Ex. $A$ III. $f(x, x, \cdots, x)=x$ when all the $x$ 's are equal; otherwise $f()=\frac{1}{2}$. This example fails on III and III', and on $A 1$ and $A 4$; but it satisfies $A 2$ and A3.

Ex. $A$ IV. $f()=\left(x_{1}+x_{2}+\cdots+x_{n}\right) / 2$.

This example fails on IV and IV', and on $A 3$ and $A 4$; but it satisfies $A 1$ and A2.

Ex. $A$ III 7. $f\left(x_{1}, x_{2}\right)=\left(x_{1}+x_{2}\right) / 2$; but when $n>2, f()=1$ or $\frac{1}{2}$, according as $\left(x_{1}+x_{2}+\cdots+x_{n}\right) / n$ is equal to 1 or not equal to 1 . This example is used only in Set $A 7$, to prove the independence of Postulate III in that set. It fails on III and IV, but satisfies III' and IV'. To see that it satisfies $A 2$ and $A 3$, note that if all the $x$ 's are less than 1 , the value of $\left(x_{1}+x_{2}+\cdots+x_{n}\right) / n$ must be less than 1 , and hence $f()=\frac{1}{2}$.

Ex. $G$ I. $f()=\left(x_{1} x_{2} x_{3}{ }^{3} x_{4}{ }^{4} \cdots x_{n}^{n}\right)^{1 / p}$, where $p=1+1+3+4+\cdots+n$.

Ex. $G$ II. $f()=$ the median of the $n$ quantities if $n$ is odd, or the geometric mean of the median-pair if $n$ is even. To see that II fails, note that $f(4,9,5)$ $=5$, while $f(6,6,5)=6$.

Ex. $G$ III. $f(x, x, \cdots, x)=x$ when all the $x$ 's are equal; otherwise $f()=1$.

Ex. G IV. $f()=\left\{\left(x_{1} x_{2} \cdots x_{n}\right) /(n-1)\right\}^{1 / n}$. To see that II holds, note that $m=f\left(x_{1}, x_{2}\right)=\left(x_{1} x_{2}\right)^{1 / 2}$, so that $m m=x_{1} x_{2}$.

Ex. G III 7. $f\left(x_{1}, x_{2}\right)=\left(x_{1} x_{2}\right)^{1 / 2}$; but when $n>2, f()=1$. This example is used only in Set G7, to prove the independence of Postulate III in that set.

Ex. $G$ V. $f(x, x, \cdots, x)=x$ when all the $x$ 's are equal; otherwise $f()=-\left|x_{1} x_{2} \cdots x_{n}\right|^{1 / n}$, where the expression of which the $n$th root is taken is the absolute value of the product of the $x$ 's without regard to sign.

Here when $n=2$,

$$
f()=\frac{1+1+3+4+\cdots+n}{\frac{1}{x_{1}}+\frac{1}{x_{2}}+\frac{3}{x_{3}}+\frac{4}{x_{4}}+\cdots+\frac{n}{x_{n}}} .
$$

$$
f\left(x_{1}, x_{2}\right)=\frac{2 x_{1} x_{2}}{x_{1}+x_{2}} .
$$

Ex. $H$ II. $f()=$ the median of the $n$ quantities if $n$ is odd, or the harmonic mean of the median-pair if $n$ is even. To see that II fails, note that $f(3,6,2)$ $=3$, while $f(4,4,2)=4$.

*Here the median (or median-pair) of $n$ positive quantities is defined by arranging the $n$ quantities in a series in order of magnitude, and picking out the middle item (or mid-pair) in this series. 
Ex. $H$ III. $f(x, x, \cdots, x)=x$ if all the $x$ 's are equal; otherwise $f()=2$.

Ex. H IV. $\quad f()=2 /\left(\frac{1}{x_{1}}+\frac{1}{x_{2}}+\cdots+\frac{1}{x_{n}}\right)$.

Ex. $H$ III 7. $f\left(x_{1}, x_{2}\right)=2 x_{1} x_{2} /\left(x_{1}+x_{2}\right)$; but when $n>2, f()=1$ or 2 , according as $(1 / n)\left[\left(1 / x_{1}\right)+\left(1 / x_{2}\right)+\cdots+\left(1 / x_{n}\right)\right]$ is equal to 1 or not equal to 1 . This example is used only in Set $H 7$, to prove the independence of Postulate III in that set.

Ex. $H$ V. $f(x, x, \cdots, x)=x$ when all the $x$ 's are equal; otherwise $f()=0$.

Ex. $S$ I.

$$
f()=\left(\frac{x_{1}^{2}+x_{2}^{2}+3 x_{3}^{2}+4 x_{4}^{2}+\cdots+n x_{n}^{2}}{1+1+3+4+\cdots+n}\right)^{1 / 2}
$$

To see that this example satisfies II, note that $m=f\left(x_{1}, x_{2}\right)=\left\{\left(x_{1}^{2}+x_{2}^{2}\right) / 2\right\}^{1 / 2}$, so that $m^{2}+m^{2}=x_{1}^{2}+x_{2}^{2}$.

Ex. $S$ II. $f()=$ the median of the $n$ quantities if $n$ is odd, or the root-meansquare of the median-pair if $n$ is even. To see that II fails, note that $f(6,8,4)$ $=6$, while $f\left(50^{1 / 2}, 50^{1 / 2}, 4\right)=50^{1 / 2}$.

Ex. $S$ III. $f(x, x, \cdots, x)=x$ when all the $x$ 's are equal; otherwise $f()=(1 / 2)^{1 / 2}$.

Ex. $S$ IV. $f()=\left\{\left(x_{1}^{2}+x_{2}^{2}+\cdots+x_{n}^{2}\right) / 2\right\}^{1 / 2}$.

Ex. $S$ III 7. $f\left(x_{1}, x_{2}\right)=\left\{\left(x_{1}^{2}+x_{2}^{2}\right) / 2\right\}^{1 / 2}$; but when $n>2, f()=1$ or $(1 / 2)^{1 / 2}$, according as $\left(x_{1}^{2}+x_{2}^{2}+\cdots+x_{n}^{2}\right) / n$ is equal to 1 or not equal to 1 . This example is used only in Set $S 7$, to prove the independence of Postulate III in that set.

\section{Proofs of THEOREMS}

In the following paragraphs, we give the proof that each of the foregoing sets of postulates is sufficient to define the type of mean in question.

Theorem A (A). Proof of A from A1, I, II, and IV.

Let $q$ be any positive quantity which is less than $(1 / n)$ th of the smallest of the $x$ 's. Then by II and $A 1$,

$$
f\left(x_{1}, x_{2}, x_{3}, \cdots, x_{n}\right)=f\left(q,\left[x_{1}+x_{2}-q\right], x_{3}, \cdots, x_{n}\right),
$$

since each side equals $f\left(\frac{1}{2}\left(x_{1}+x_{2}\right), \frac{1}{2}\left(x_{1}+x_{2}\right), x_{3}, \cdots, x_{n}\right)$, and all the arguments are positive.

By successive applications of this result, in view of $I$, we have

$$
\begin{aligned}
f\left(x_{1}, x_{2}, \cdots, x_{n}\right) & =f\left(q, q,\left[x_{1}+x_{2}+x_{3}-2 q\right], x_{4}, \cdots, x_{n}\right) \\
& =f\left(q, q, q,\left[x_{1}+x_{2}+x_{3}+x_{4}-3 q\right], x_{5}, \cdots, x_{n}\right) \\
& =f\left(q, q, \cdots,\left[x_{1}+x_{2}+\cdots+x_{n}-(n-1) q\right]\right) .
\end{aligned}
$$


Now take $a=\left(x_{1}+x_{2}+\cdots+x_{n}\right) / n$. Then, putting each $x$ equal to $a$, $f(a, a, \cdots, a)=f(q, q, \cdots,[n a-(n-1) q])=a$, by Postulate IV. But $x_{1}+x_{2}+\cdots+x_{n}=n a$, so that

Hence

$$
f\left(x_{1}, x_{2}, \cdots, x_{n}\right)=f(q, q, \cdots,[n a-(n-1) q]) .
$$

$$
f\left(x_{1}, x_{2}, \cdots, x_{n}\right)=\left(x_{1}+x_{2}+\cdots+x_{n}\right) / n .
$$

Hence any function $f$ which satisfies the postulates of Set $A 1$ must be identical with the arithmetic mean, $A$.

Theorem A (B). Proof of $A 1$ from $A 2$, I, and III or III'.

$$
\begin{aligned}
f(a, b) & =(a+b) f\left(\frac{a}{a+b}, \frac{b}{a+b}\right), \text { by III or III'; } \\
& =(a+b) f\left(1-\frac{b}{a+b}, 1-\frac{a}{a+b}\right) \\
& =(a+b)\left[1-f\left(\frac{b}{a+b}, \frac{a}{a+b}\right)\right], \text { by } A 2 ; \\
& =(a+b)-f(b, a), \text { by III or } \mathrm{III}^{\prime} ; \\
& =(a+b)-f(a, b), \text { by I. }
\end{aligned}
$$

Hence $f(a, b)=(a+b) / 2$, which is $A 1$.

This proof shows that any function which satisfies the postulates of Set $A 2$ or Set $A 5$ will also satisfy the postulates of Set $A 1$, and hence be identical with the arithmetic mean.

Theorem A (c). Proof of IV from A3, III.

From $A 3$, putting $x_{1}=\frac{1}{2}, x_{2}=\frac{1}{2}, \cdots, x_{n}=\frac{1}{2}$, we have

$$
f\left(\frac{1}{2}, \frac{1}{2}, \cdots, \frac{1}{2}\right)=1-f\left(\frac{1}{2}, \frac{1}{2}, \cdots, \frac{1}{2}\right) \text {, }
$$

whence $f\left(\frac{1}{2}, \frac{1}{2}, \cdots, \frac{1}{2}\right)=\frac{1}{2}$. Hence by III, $f(a, a, \cdots, a)=a$, which is IV.

This proof shows (since Postulate $A 2$ follows at once from Postulate $A 3$ ) that any function which satisfies Set $A 3$ will also satisfy Set $A 2$.

Theorem A (D). Proof of $A 1$ and IV from A4, I.

From $A 4$, putting $A=a+b, x_{1}=a, x_{2}=b$, and $n=2$, we have

$$
f(a+b-a, a+b-b)=a+b-f(a, b) ;
$$

whence by I, $f(a, b)=a+b-f(a, b)$. Therefore $f(a, b)=(a+b) / 2$, which is $A 1$. 
Again, from $A 4$, putting each $x$ equal to $a$, and $A=2 a$, we have

$$
f(a, a, \cdots, a)=2 a-f(a, a, \cdots, a) \text {, }
$$

whence $f(a, a, \cdots, a)=a$, which is IV.

This proof shows that any function which satisfies Set $A 4$ will also satisfy Set $A 1$.

Theorem A (E). Since IV follows from III and IV', we see that Set $A 6$ implies Set $A 2$, and that Set $A 7$ implies Set $A 1$; so that any function which satisfies Set $A 6$ or Set $A 7$ will be identical with the arithmetic mean.

Theorem G (A). Proof of $G$ from G1, I, II, IV.

By II and $G 1, f\left(x_{1}, x_{2}, x_{3}, \cdots, x_{n}\right)=f\left(1,\left[x_{1} x_{2}\right], x_{3}, \cdots, x_{n}\right)$, since each side equals $f\left\{\left(x_{1} x_{2}\right)^{1 / 2},\left(x_{1} x_{2}\right)^{1 / 2}, x_{3}, \cdots, x_{n}\right\}$.

By successive applications of this result, in view of $I$, we have

$$
\begin{aligned}
& f\left(x_{1}, x_{2}, \cdots, x_{n}\right)=f\left(1,1,\left[x_{1} x_{2} x_{3}\right], x_{4}, \cdots, x_{n}\right) \\
& =f\left(1,1,1,\left[x_{1} x_{2} x_{3} x_{4}\right], x_{5}, \cdots, x_{n}\right) \\
& =f\left(1,1, \cdots,\left[x_{1} x_{2} \cdots x_{n}\right]\right) .
\end{aligned}
$$

Now take $a=\left(x_{1} x_{2} \cdots x_{n}\right)^{1 / n}$. Then, putting each $x$ equal to $a$, we see that $f(a, a, \cdots, a)=f\left(1,1, \cdots, a^{n}\right)=a$, by Postulate IV. But $x_{1} x_{2} \cdots x_{n}=a^{n}$, so that $f\left(x_{1}, x_{2}, \cdots, x_{n}\right)=f\left(1,1, \cdots, a^{n}\right)=a$. Hence

$$
f\left(x_{1}, x_{2}, \cdots, x_{n}\right)=\left(x_{1} x_{2} \cdots x_{n}\right)^{1 / n} \text {. }
$$

This proof shows that Set $G 1$ determines the geometric mean.

Theorem G (B). Proof of $G 1$ from G2, I, III or III', V.

$$
\begin{aligned}
f(a, b) & =a b f\left(\frac{1}{b}, \frac{1}{a}\right), \text { by III or III', } \\
& =a b \frac{1}{f(b, a)}, \text { by } G 2 \\
& =a b \frac{1}{f(a, b)}, \text { by I. }
\end{aligned}
$$

Hence $[f(a, b)]^{2}=a b$, so that $f(a, b)=(a b)^{1 / 2}$ or $-(a b)^{1 / 2}$. But the negative value is impossible, by V. Hence $f(a, b)=(a b)^{1 / 2}$, which is $G 1$.

This proof shows that Set $G 2$ and Set $G 5$ reduce to Set $G 1$, and hence determine the geometric mean.

Theorem G (c). Proof of IV from G3, III, V. 
From $G 3$, putting $x_{1}=1, x_{2}=1, \cdots, x_{n}=1$, we have $f(1,1, \cdots, 1)$ $=1 / f(1,1, \cdots, 1)$, whence $f(1,1, \cdots, 1)=1$ or -1 . Hence by $\mathbf{V}$, $f(1,1, \cdots, 1)=1$. Then by III, $f(a, a, \cdots, a)=a$, which is IV.

This proof shows (since $G 2$ follows at once from $G 3$ ) that Set $G 3$ reduces to Set $G 2$, and hence determines the geometric mean.

Theorem G (D). Proof of G1 and IV from G4, I, V.

From $G 4$, putting $a=a b, x_{1}=a, x_{2}=b$, and $n=2$, we have

Hence by I,

$$
f\left(\frac{a b}{a}, \frac{a b}{b}\right)=\frac{a b}{f(a, b)} .
$$

$$
f(a, b)=\frac{a b}{f(a, b)} .
$$

Therefore $\left.f^{\prime} a, b\right)=(a b)^{1 / 2}$ or $-(a b)^{1 / 2}$. But the negative value is excluded, by V. Hence $f(a, b)=(a b)^{1 / 2}$, which is $G 1$.

Again, from $G 4$, putting $x$ equal to $a$, and $A=a^{2}$, we have

$$
f(a, a, \cdots, a)=\frac{a^{2}}{f(a, a, \cdots, a)}
$$

whence, by V, $f(a, a, \cdots, a)=a$, which is IV.

This proof shows that Set $G 4$ reduces to Set $G 1$, and hence determines the geometric mean.

Theorem $G(E)$. Since IV follows at once from III and $I^{\prime}$, we see that Set $G 6$ reduces to Set $G 2$, and that Set $G 7$ reduces to Set $G 1$; hence Set $G 6$ and Set $G 7$ determine the geometric mean.

Theorem H (A). Proof of $H$ from $H 1$, I, II, IV.

Let $q$ be any positive quantity which is greater than $n$ times the largest of the $x$ 's. Then by II and $H 1$,

$$
f\left(x_{1}, x_{2}, \cdots, x_{n}\right)=f\left(q, \frac{1}{\frac{1}{x_{1}}+\frac{1}{x_{2}}-\frac{1}{q}}, x_{3}, \cdots, x_{n}\right),
$$

since each side is equal to

$$
f\left(\frac{2 x_{1} x_{2}}{x_{1}+x_{2}}, \frac{2 x_{1} x_{2}}{x_{1}+x_{2}}, x_{3}, \cdots, x_{n}\right)
$$


and all the arguments are positive. By successive applications of this result, using I, we have

$$
\begin{aligned}
f\left(x_{1}, x_{2}, \cdots, x_{n}\right) & =f\left(q, q, \frac{1}{\frac{1}{x_{1}}+\frac{1}{x_{2}}+\frac{1}{x_{3}}-\frac{2}{q}}, x_{4}, \cdots, x_{n}\right) \\
& \left.\cdot \cdot \cdot \cdot \cdot \cdot \frac{\cdot}{\frac{1}{x_{1}}+\frac{1}{x_{2}}+\cdots+\frac{1}{x_{n}}-\frac{n-1}{q}}\right) .
\end{aligned}
$$

Now take

$$
a=\left(\frac{n}{\frac{1}{x_{1}}+\frac{1}{x_{2}}+\cdots+\frac{1}{x_{n}}}\right) .
$$

Then putting each $x$ equal to $a$,

$$
f(a, a, \cdots, a)=\left(q, q, \cdots, \frac{1}{\frac{n}{a}-\frac{n-1}{q}}\right)=a,
$$

by Postulate IV. But

$$
\frac{1}{x_{1}}+\frac{1}{x_{2}}+\cdots+\frac{1}{x_{n}}=\frac{n}{a}
$$

so that

$$
f\left(x_{1}, x_{2}, \cdots, x_{n}\right)=f\left(q, q, \cdots, \frac{1}{\frac{n}{a}-\frac{n-1}{q}}\right)=a .
$$

Hence

$$
f\left(x_{1}, x_{2}, \cdots, x_{n}\right)=\frac{1}{\frac{1}{n}\left(\frac{1}{x_{1}}+\frac{1}{x_{2}}+\cdots+\frac{1}{x_{n}}\right)} .
$$

This proof shows that Set $B 1$ determines the harmonic mean. 
TheOREM H (B). Proof of $H 1$ from H2, I, III or III', V.

$$
\begin{aligned}
f(a, b) & =\frac{a b}{a+b} f\left(\frac{a+b}{b}, \frac{a+b}{a}\right), \quad \text { by III or } \mathrm{III}^{\prime} ; \\
& =\frac{a b}{a+b} f\left(\frac{\frac{a+b}{a}}{\frac{a+b}{a}-1}, \frac{\frac{a+b}{b}}{\frac{a+b}{b}-1}\right) \\
& =\frac{a b}{a+b} \frac{f\left(\frac{a+b}{a}, \frac{a+b}{b}\right)}{f\left(\frac{a+b}{a}, \frac{a+b}{b}\right)-1}, \quad \text { by } H 2 .
\end{aligned}
$$

Now by $\mathrm{V}, f(a, b)$ is not zero. Hence

$$
\begin{aligned}
\frac{1}{f(a, b)} & =\frac{1}{\frac{a b}{a+b}}-\frac{1}{\frac{a b}{a+b} f\left(\frac{a+b}{a}, \frac{a+b}{b}\right)} \\
& =\frac{a+b}{a b}-\frac{1}{f(b, a)}, \text { by III or } \mathrm{III}^{\prime} ; \\
& =\frac{a+b}{a b}-\frac{1}{f(a, b)}, \text { by I. }
\end{aligned}
$$

Hence

$$
f(a, b)=\frac{2 a b}{a+b},
$$

which is $H 1$.

This proof shows that Set $B 2$ and Set $B 5$ reduce to Set $B 1$, and hence determine the harmonic mean.

Theorem H (c). Proof of IV from H3, III, V.

From $H 3$, putting $x_{1}=2, x_{2}=2, \cdots, x_{n}=2$, we have

$$
f(2,2, \cdots, 2)=\frac{f(2,2, \cdots, 2)}{f(2,2, \cdots, 2)-1} .
$$

Hence $f(2,2, \cdots, 2)[f(2,2, \cdots, 2)-2]=0$. But by $\mathrm{V}, f(2,2, \cdots, 2)$ is not zero. Hence $f(2,2, \cdots, 2)=2$. Hence by $\operatorname{III}, f(a, a, \cdots, a)=a$, which is IV. 
This proof shows (since $H 2$ follows at once from $H 3$ ) that Set $H 3$ reduces to Set $H 2$, and hence determines the harmonic mean.

Theorem H (D). Proof of $H 1$ and IV from $H 4$.

From $H 4$, putting $A=(a+b) /(a b), x_{1}=a, x_{2}=a$, and $n=2$, we have

whence by $\mathrm{I}$,

$$
f(b, a)=\frac{(a b) f(a, b)}{(a+b) f(a, b)-a b},
$$

$$
f(a, b)=\frac{(a b) f(a, b)}{(a+b) f(a, b)-a b}
$$

where by V, $f(a, b)$ is not zero; hence $f(a, b)=2 a b /(a+b)$, which is $H 1$.

Again, from $H 4$, putting each $x$ equal to $a$, and $A=2 / a$, we have

$$
f(a, a, \cdots, a)=\frac{a f(a, a, \cdots, a)}{2 f(a, a, \cdots, a)-a},
$$

where by V, $f(a, a, \cdots, a)$ is not zero; hence $f(a, a, \cdots, a)=a$, which is IV.

This proof shows that Set $H 4$ reduces to Set $H 1$, and hence determines the harmonic mean.

THEOREM H (E). Since IV follows at once from III and IV', we see that Set $H 6$ and Set $H 7$ reduce to Set $H 2$ and Set $H 1$ respectively, and hence determine the harmonic mean.

Theorem S (A). Proof of $S$ from $S 1, \mathrm{I}, \mathrm{II}, \mathrm{IV}$.

Let $q$ be any positive quantity which is smaller than $(1 / n)$ th of the smallest of the $x$ 's. Then by II and $S 1$,

$$
f\left(x_{1}, x_{2}, x_{3}, \cdots, x_{n}\right)=f\left\{q,\left(x_{1}^{2}+x_{2}^{2}-q^{2}\right)^{1 / 2}, x_{3}, \cdots, x_{n}\right\},
$$

since each side is equal to $f\left\{\left[\left(x_{1}^{2}+x_{2}^{2}\right) / 2\right]^{1 / 2},\left[\left(x_{1}^{2}+x_{2}^{2}\right) / 2\right]^{1 / 2}, x_{3}, \cdots, x_{n}\right\}$, and all the arguments are positive. By successive applications of this result, in view of $I$, we have

$$
\begin{aligned}
f\left(x_{1}, x_{2}, \cdots x_{n}\right)= & f\left\{q, q,\left(x_{1}^{2}+x_{2}^{2}+x_{3}^{2}-2 q^{2}\right)^{1 / 2}, x_{4}, \cdots x_{n}\right\} \\
& \cdot \cdot \cdot \cdot \cdot \cdot \cdot \\
& =f\left\{q, q, \cdots,\left(x_{1}^{2}+x_{2}^{2}+\cdots+x_{n}^{2}-(n-1) q^{2}\right)^{1 / 2}\right\} .
\end{aligned}
$$

Now take $a=\left\{\left(x_{1}^{2}+x_{2}^{2}+\cdots+x_{n}^{2}\right) / n\right\}^{1 / 2}$. Then putting each $x$ equal to $a$, $f(a, a, \cdots, a)=f\left\{q, q, \cdots,\left(n a^{2}-(n-1) q^{2}\right)^{1 / 2}\right\}=a$, by IV.

But $x_{1}^{2}+x_{2}^{2}+\cdots+x_{n}^{2}=n a^{2}$, so that

$$
f\left(x_{1}, x_{2}, \cdots, x_{n}\right)=f\left\{q, q, \cdots,\left(n a^{2}-(n-1) q^{2}\right)^{1 / 2}\right\}=a .
$$


Hence

$$
f\left(x_{1}, x_{2}, \cdots, x_{n}\right)=\left(\frac{x_{1}{ }^{2}+x_{2}^{2}+\cdots+x_{n}^{2}}{n}\right)^{1 / 2} .
$$

This proof shows that Set $S 1$ determines the root-mean-square.

Theorem S (в). Proof of S1 from S2, I, III or III'.

$$
\begin{aligned}
f(a, b) & =\left(a^{2}+b^{2}\right)^{1 / 2} f\left(\frac{a}{\left(a^{2}+b^{2}\right)^{1 / 2}}, \frac{b}{\left(a^{2}+b^{2}\right)^{1 / 2}}\right), \quad \text { by III or III' } ; \\
& \left.=\left(a^{2}+b^{2}\right)^{1 / 2} f\left(1-\frac{b^{2}}{a^{2}+b^{2}}\right)^{1 / 2},\left(1-\frac{a^{2}}{a^{2}+b^{2}}\right)^{1 / 2}\right] \\
& =\left(a^{2}+b^{2}\right)^{1 / 2}\left(1-\left[f\left(\frac{b}{\left(a^{2}+b^{2}\right)^{1 / 2}}, \frac{a}{\left(a^{2}+b^{2}\right)^{1 / 2}}\right]^{2}\right)^{1 / 2} \text { by } S 2 ;\right. \\
& =\left\{\left(a^{2}+b^{2}\right)-[f(b, a)]^{2}\right\}^{1 / 2}, \text { by III or III' } \\
& =\left\{\left(a^{2}+b^{2}\right)-[f(a, b)]^{2}\right\}^{1 / 2} \text { by I. }
\end{aligned}
$$

Hence $2[f(a, b)]^{2}=a^{2}+b^{2}$, so that

$$
f(a, b)=\left\{\left(a^{2}+b^{2}\right) / 2\right\}^{1 / 2} \text { or }-\left\{\left(a^{2}+b^{2}\right) / 2\right\}^{1 / 2} \text {. }
$$

It remains to exclude the negative value (without using V). By $S 2, f(a, b)$ is positive whenever $a<1$ and $b<1$; hence by III, $f(a, b)$ is positive for all positive values of $a$ and $b$. Hence $f(a, b)=\left\{\left(a^{2}+b^{2}\right) / 2\right\}^{1 / 2}$.

This proof shows that Set $S 2$ and Set $S 5$ reduce to Set $S 1$, and hence determine the root-mean-square.

Theorem S (c). Proof of IV from S3, III.

From $S 3$, putting $x_{1}=\frac{1}{2}, x_{2}=\frac{1}{2}, \cdots, x_{n}=\frac{1}{2}$, we have

$$
\begin{aligned}
& f\left\{(1 / 2)^{1 / 2},(1 / 2)^{1 / 2}, \cdots,(1 / 2)^{1 / 2}\right\} \\
& =\left(1-\left[f\left\{(1 / 2)^{1 / 2},(1 / 2)^{1 / 2}, \cdots,(1 / 2)^{1 / 2}\right\}\right]^{2}\right)^{1 / 2}
\end{aligned}
$$

which by definition of the square root sign, is not negative. Hence

Hence by III,

$$
f\left\{(1 / 2)^{1 / 2},(1 / 2)^{1 / 2}, \cdots,(1 / 2)^{1 / 2}\right\}=(1 / 2)^{1 / 2}
$$

$$
f(a, a, \cdots, a)=a .
$$

This proof shows (since $S 2$ follows at once from $S 3$ ) that Set $S 3$ reduces to Set $S 2$, and hence determines the root-mean-square.

TheOREM S (D). Proof of $S 1$ and IV from S4, I.

From $S 4$, putting $A=a^{2}+b^{2}, x_{1}=a, x_{2}=b$, and $n=2$, we have

$$
f(b, a)=\left(a^{2}+b^{2}-[f(a, b)]^{2}\right)^{1 / 2} .
$$

Hence, by $\mathrm{I}, f(a, b)=\left(a^{2}+b^{2}-[f(a, b)]^{2}\right)^{1 / 2}$, which is not negative. Hence $f(a, b)=\left\{\left(a^{2}+b^{2}\right) / 2\right\}^{1 / 2}$. 
Again, from $S 4$, putting $x$ equal to $a$, and $A=2 a^{2}$, we have $f(a, a, \cdots, a)=\left\{2 a^{2}-[f(a, a, \cdots, a)]^{2}\right\}^{1 / 2}$, which is not negative; hence $f(a, a, \cdots, a)=a$, which is Postulate IV.

This proof shows that Set $S 4$ reduces to Set $S 1$, and hence determines the root-mean-square.

Theorem S (E). Since IV follows at once from III and IV', we see that Set $S 6$ and Set $S 7$ reduce to Set $S 2$ and Set $S 1$ respectively, and hence determine the root-mean-square.

Appendix I. The arithmetic MEAN IN THE DOMAIN OF ALL REAL NUMBERS

In all the preceding sets of postulates, the $x$ 's in the function $f\left(x_{1}, x_{2}\right.$, $\cdots, x_{n}$ ) have been assumed to be positive real quantities. In the case of the geometric mean, the harmonic mean, and the root-mean-square, this restriction is a customary one, in order to insure that the function shall always be finite and single-valued. In the case of the arithmetic mean, however, the restriction is not essential.

This appendix, therefore, is devoted to a consideration of sets of postulates for the arithmetic mean in the domain of all real numbers.

For this purpose, we introduce, in addition to the postulates $\mathrm{I}-\mathrm{V}, \mathrm{III}^{\prime}$, $\mathrm{IV}^{\prime}, A 1-A 4$, given above, the following postulates:

Postulate IV". $f(-1,-1, \cdots,-1)=-1$.

Postulate $A 8$. $f\left(A+x_{1}, A+x_{2}, \cdots, A+x_{n}\right)=A+f\left(x_{1}, x_{2}, \cdots, x_{n}\right)$.

Postulate $A 8^{\prime} . \quad f\left(-x_{1},-x_{2}, \cdots,-x_{n}\right)=-f\left(x_{1}, x_{2}, \cdots, x_{n}\right)$.

The results obtained may be summarized as follows:

In the first place, Sets $A 1, A 2, A 3, A 4, A 5$, given above for the case of positive reals, are valid just as they stand for the case of all reals. (The necessary modifications in the proofs are given below.)

In the second place, Sets $A 6$ and $A 7$ are not valid for the case of all reals (see Example $A$ IV $^{\prime \prime}$ below); but they can be made so by the addition of Postulate IV'.

In the third place, Postulates I, II, $A 8, A 8^{\prime}$ form a set (due essentially to Schimmack*), which is valid for the case of all reals, but cannot be used in the case of positive reals; this we shall call Set $A 8$.

\footnotetext{
*Instead of our Postulate II, Schimmack (loc. cit.) uses the following postulate: $f\left(x_{1}, x_{2}, \cdots, x_{n-1}, x_{n}\right)=f\left(m, m, \cdots, m, x_{n}\right)$, where $m=f\left(x_{1}, x_{2}, \cdots, x_{n-1}\right)$; and instead of our Example $A \mathrm{I}$, the following example: $f()=\left(x_{1} / 2^{n-1}\right)+\left(x_{2} / 2^{n-1}\right)+\left(x_{3} / 2^{n-2}\right)+\left(x_{4} / 2^{n-3}\right)+\cdots+\left(x_{n-1} / 2^{2}\right)+\left(x_{n} / 2\right)$.
} 
The complete list of sets of independent postulates for the arithmetic mean, for the case of all reals, is then as follows:

\begin{tabular}{|c|c|c|c|c|c|c|c|c|c|c|c|}
\hline Set & \multicolumn{6}{|c|}{ Postulates } & \multicolumn{5}{|c|}{ Examples Used } \\
\hline$A 1$ & $A 1$ & I & II & & IV & & $0 \quad A \mathrm{I}$ & $A \mathrm{II}^{\prime}$ & & $A \mathrm{IV}$ & \\
\hline$A 2$ & $A 2$ & I & II & III & IV & & $0 \quad A \mathrm{I}$ & $A \mathrm{II}^{\prime}$ & $A$ III & $A \mathrm{IV}$ & \\
\hline$A 3$ & $A 3$ & I & II & III & & & $0 \quad A \mathrm{I}$ & $A \mathrm{II}^{\prime}$ & $A$ IIII & & \\
\hline$A 4$ & $A 4$ & I & II & & & & $0 A \mathrm{I}$ & $A \mathrm{II}^{\prime}$ & & & \\
\hline$A 5$ & $A 2$ & I & II & III' $^{\prime}$ & IV & & $0 A \mathrm{I}$ & $A \mathrm{II}^{\prime}$ & $A$ III & $A \mathrm{IV}$ & \\
\hline$A 6^{\prime \prime}$ & $A 2$ & I & II & III & IV $^{\prime}$ & IV ${ }^{\prime \prime}$ & $0 \quad A \mathrm{I}$ & $A \mathrm{II}^{\prime}$ & $A \mathrm{III}^{\prime \prime}$ & $A \mathrm{IV}^{\prime}$ & $A \mathrm{IV}^{\prime \prime}$ \\
\hline$A 7^{\prime \prime}$ & $A 1$ & I & II & III & $\mathrm{IV}^{\prime}$ & $I V^{\prime \prime}$ & $0 A \mathrm{I}$ & $A \mathrm{II}^{\prime}$ & $A \mathrm{III}^{\prime \prime}$ & $A \mathrm{IV}^{\prime}$ & $A \mathrm{IV}^{\prime \prime}$ \\
\hline$A 8$ & $A 8$ & I & II & $A 8^{\prime}$ & & & $\begin{array}{ll}0 & A \mathrm{I} \\
\end{array}$ & $A \mathrm{II}^{\prime}$ & $0^{\prime}$ & & \\
\hline
\end{tabular}

The new examples in the independence proofs are the following:

Ex. $A \mathrm{II}^{\prime}$. The same as Ex. $A$ II, for all reals, understanding "the order of magnitude" in the algebraic sense.

Ex. $A$ III' $^{\prime \prime}$. When $n=2, f\left(x_{1}, x_{2}\right)=\frac{1}{2}\left(x_{1}+x_{2}\right)$; when $n>2$,

$$
f\left(x_{1}, x_{2}, \cdots, x_{n}\right)=1 \text { or }-1
$$

according as $\left(x_{1}+x_{2}+\cdots+x_{n}\right) / n$ is equal to 1 or not equal to 1 .

Ex. $A \mathrm{IV}^{\prime}$. When $n=2, f\left(x_{1}, x_{2}\right)=\frac{1}{2}\left(x_{1}+x_{2}\right)$; when $n>2$,

$$
f()=-\left|\left(x_{1}+x_{2}+\cdots+x_{n}\right) / n\right|,
$$

where the vertical bars mean "the absolute value of."

Ex. $A \mathrm{IV}^{\prime \prime}$. When $n=2, f\left(x_{1}, x_{2}\right)=\frac{1}{2}\left(x_{1}+x_{2}\right)$; when $n>2$,

$$
f()=\left|\left(x_{1}+x_{2}+\cdots+x_{n}\right) / n\right| .
$$

Ex. $0^{\prime} . f()=$ the maximum (in the algebraic sense) of the $n$ real quantities $x_{1}, x_{2}, \cdots, x_{n}$.

\begin{tabular}{|c|c|c|c|c|c|c|c|c|c|c|c|c|c|}
\hline & $A 1$ & $A 2$ & $A 3$ & $A 4$ & $A 8$ & $A 8^{\prime}$ & I & II & III & IV & III' & IV & $\mathrm{IV}^{\prime \prime}$ \\
\hline Ex. 0 & - & - & - & 一 & 一 & $A 8^{\prime}$ & I & II & III & IV & III' & IV & $I^{\prime \prime}$ \\
\hline Ex. $0^{\prime}$ & - & - & - & - & $A 8$ & - & I & II & III & IV & III' & IV & $I V^{\prime \prime}$ \\
\hline Ex. $A$ I & $A 1$ & $A 2$ & $A 3$ & $A 4$ & $A 8$ & $A 8^{\prime}$ & - & II & III & IV & III' $^{\prime}$ & IV & $I V^{\prime \prime}$ \\
\hline Ex. $A \mathrm{II}^{\prime}$ & $A 1$ & $A 2$ & $A 3$ & $A 4$ & $A 8$ & $A 8^{\prime}$ & I & - & III & IV & III' $^{\prime}$ & IV & $I V^{\prime \prime}$ \\
\hline Ex. $A$ III & - & $A 2$ & $A 3$ & 一 & 一 & - & I & II & 一 & IV & - & IV & IV" \\
\hline Ex. $A$ IV & $A 1$ & $A 2$ & - & 一 & - & $A 8^{\prime}$ & I & II & III & - & III' & - & - \\
\hline Ex. $A \mathrm{III}^{\prime \prime}$ & $A 1$ & $A 2$ & - & - & 一 & - & I & II & - & - & III $^{\prime}$ & IV & $I^{\prime \prime}$ \\
\hline Ex. $A \mathrm{IV}^{\prime}$ & $A 1$ & $A 2$ & - & 一 & - & - & I & II & III & - & III' $^{\prime}$ & - & IV" \\
\hline Ex. $A \mathrm{IV}^{\prime \prime}$ & $A 1$ & $A 2$ & - & - & - & - & I & II & III & - & III' $^{\prime}$ & IV $^{\prime}$ & - \\
\hline
\end{tabular}

The following table shows the properties of all the examples used in the case of the real domain. 
The proofs of the theorems, adapted to the domain of all reals, are as follows:

Theorem A $\left(\mathrm{A}^{\prime}\right)$. Proof of $A$ from $A 1, \mathrm{I}, \mathrm{II}, \mathrm{IV}$. (Real domain.)

Since there is now no necessity for keeping the arguments positive, the proof of Theorem A (A) can be simplified by putting $q=0$. Hence Set $A 1$ determines the arithmetic mean.

Theorem A (B'). Proof of A1 from A2, I, III or III'. (Real domain.)

If $a$ and $b$ are positive, $f(a, b)=\frac{1}{2}(a+b)$, as in the proof of Theorem A (B).

If $a$ and $b$ are any real numbers, let $p=1 /(|a|+|b|+1)$; then $1-p a$ and $1-p b$ will be positive, so that

$$
f(1-p a, 1-p b)=\frac{1}{2}(1-p a+1-p b)=1-p(a+b) / 2 \text {. }
$$

But $f(1-p a, 1-p b)=1-f(p a, p b)$, by $A 2 ;=1-p f(a, b)$, by III or III'. Therefore $f(a, b)=(a+b) / 2$, for all real values of $a$ and $b$. Hence Set $A 2$ and Set $A 5$ reduce to Set $A 1$.

Theorem A $\left(\mathrm{c}^{\prime}\right)$. Proof of IV from A3, I, III. (Real domain.)

By III, $f(0,0, \cdots, 0)=2 f(0,0, \cdots, 0)$; hence $f(0,0, \cdots, 0)=0$.

From $A 3, f(1,1, \cdots, 1)=1-f(0,0, \cdots, 0)=1$. Hence by III,

whenever $a$ is positive.

$$
f(a, a, \cdots, a)=a \text {, }
$$

From $A 3, f(2,2, \cdots, 2)=1-f(-1,-1, \cdots,-1)$, whence

$$
2=1-f(-1,-1, \cdots,-1) \text {, }
$$

whence $f(-1,-1, \cdots,-1)=-1$. Hence by III,

whenever $a$ is negative.

$$
f(a, a, \cdots, a)=a
$$

Since $A 2$ follows immediately from $A 3$, this proof shows that Set $A 3$ reduces to Set $A 2$.

Theorem A ( $\left.\mathrm{D}^{\prime}\right)$. Proof of $A 1$ and IV from A4, I, II. (Real domain.)

If $a$ and $b$ are positive, put $A=a+b, x_{1}=a, x_{2}=b$, and $n=2$, in $A 4$; then $f(b, a)=a+b-f(a, b)$, whence by I, $f(a, b)=a+b-f(a, b)$, whence

whenever $a$ and $b$ are positive.

$$
f(a, b)=\frac{1}{2}(a+b),
$$

If $a$ and $b$ are any reals, let $p=$ a large positive quantity, so that $p+a$, $p+b$, and $p+a+b$ will certainly be positive. Putting $A=p+a+b, x_{1}=a$, $x_{2}=b$, and $n=2$, in $A 4$, we have

$$
f(p+b, p+a)=p+a+b-f(a, b),
$$


whence

$$
(p+b+p+a) / 2=p+a+b-f(a, b),
$$

whence $f(a, b)=(a+b) / 2$, which is Postulate $A 1$ for all real values of $a$ and $b$.

If $a$ is positive, put $x_{1}=a, x_{2}=a, \cdots, x_{n}=a$, and $A=2 a$, in $A 4$; then $f(a, a, \cdots, a)=2 a-f(a, a, \cdots, a)$, whence $f(a, a, \cdots, a)=a$.

Again, if $a$ is positive, put $x_{1}=0, x_{2}=0, \cdots, x_{n}=0, A=a$, in $A 4$; then $f(a, a, \cdots, a)=a-f(0,0, \cdots, 0)$, whence $f(0,0, \cdots, 0)=0$.

If $a$ is negative, put $x_{1}=a, x_{2}=a, \cdots, x_{n}=a$, and $A=0$, in $A 4$; then $f(-a,-a, \cdots,-a)=-f(a, a, \cdots, a)$, whence, since $-a$ is positive, $-a=-f(a, a, \cdots, a)$; hence $f(a, a, \cdots, a)=a$.

Therefore $f(a, a, \cdots, a)=a$ for all real values of $a$, which is Postulate IV.

This proof shows that Set $A 4$ reduces to Set $A 1$.

Theorem A $\left(E^{\prime}\right)$. Since IV follows at once from III, IV', IV', we see that Set $A 6^{\prime \prime}$ and Set $A 7^{\prime \prime}$ reduce to Sets $A 2$ and $A 1$ respectively, in the domain of all reals.

Theorem A $\left(\mathrm{F}^{\prime}\right)$. Proof of $A 4$ from $A 8, A 8^{\prime}$. (Real domain.)

By $A 8, f\left(A-x_{1}, A-x_{2}, \cdots, A-x_{n}\right)=A+f\left(-x_{1},-x_{2}, \cdots,-x_{n}\right)$, whence, by $A 8^{\prime}, f\left(A-x_{1}, A-x_{2}, \cdots, A-x_{n}\right)=A-f\left(x_{1}, x_{2}, \cdots, x_{n}\right)$. This shows that Set $A 8$ reduces to Set $A 4$, and hence determines the arithmetic mean in the domain of reals. Unlike the other sets, however, this Set $A 8$ cannot be used if the $n$ quantities $x_{1}, x_{2}, \cdots, x_{n}$ are restricted to the domain of positive values.

\section{ApPendix II. Postulates for the ARIthmetic MEAN IN THE COMPLEX DOMAIN}

In the domain of complex quantities, Sets $A 1$ and $A 8$ are sufficient to determine the arithmetic mean. (See proofs below.) Further, if we form a new postulate

Postulate $A 9$. $f\left(A-x_{1}, A-x_{2}, \cdots, A-x_{n}\right)=A-f\left(x_{1}, x_{2}, \cdots, x_{n}\right)$, then a Set $A 9$, comprising Postulates I, II, $A 9$, will also be sufficient, as proved below. (This postulate $A 9$ is the same as $A 4$ without the restriction $x_{i}<1$.)

Hence, in the complex domain, we have three sets of independent postulates for the arithmetic mean, as follows:

\begin{tabular}{|c|c|c|c|c|c|c|c|c|}
\hline Set & \multicolumn{4}{|c|}{ Postulates } & \multicolumn{4}{|c|}{ Examples Used } \\
\hline$A 1$ & $A 1$ & I & II & IV & 0 & $A \mathrm{I}$ & $A \mathrm{II}^{\prime \prime}$ & $A \mathrm{IV}$ \\
\hline$A 8$ & $A 8$ & I & II & $A 8^{\prime}$ & 0 & $A \mathrm{I}$ & $A \mathrm{II}^{\prime \prime}$ & $0^{\prime}$ \\
\hline$A 9$ & $A 9$ & I & II & & 0 & $A \mathrm{I}$ & $A \mathrm{II}^{\prime \prime}$ & \\
\hline
\end{tabular}


The new example here required is

Ex. $A \mathrm{II}^{\prime \prime} . f\left(x_{1}+i y_{1}, x_{2}+i y_{2}, \cdots, x_{n}+i y_{n}\right)=x+i y$, where $x$ is the median of the $x$ 's if $n$ is odd (or the arithmetic mean of their median-pair if $n$ is even), and $y$ is the median of the $y$ 's if $n$ is odd (or the arithmetic mean of their median-pair if $n$ is even).

This example satisfies Postulates $A 1, A 8, A 8^{\prime}, A 9$, I and IV, but fails on Postulate II.

The properties of all these examples for the complex domain are shown in the following table:

\begin{tabular}{|l|cccc|ccc|}
\hline & $A 1$ & $A 8$ & $A 8^{\prime}$ & $A 9$ & I & II & IV \\
\hline Ex. 0 & $A 1$ & - & $A 8^{\prime}$ & - & I & II & IV \\
Ex. 0 & $A 1$ & $A 8$ & - & - & I & II & IV \\
\hline Ex. $A$ I & $A 1$ & $A 8$ & $A 8^{\prime}$ & $A 9$ & - & II & IV \\
Ex. $A$ II' & $A 1$ & $A 8$ & $A 8^{\prime}$ & $A 9$ & I & - & IV \\
Ex. $A$ IV & $A 1$ & - & $A 8^{\prime}$ & - & I & II & - \\
\hline
\end{tabular}

On the other hand, it is interesting to note that if the $n$ quantities $x_{1}, x_{2}, \cdots, x_{n}$ are allowed to take on complex values, then Sets $A 2, A 3, A 4$, $A 5, A 6, A 7, A 6^{\prime \prime}, A 7^{\prime \prime}$ are not sufficient to determine the arithmetic mean, as is shown by the following examples:

Ex. $A$ IX.

$f\left(x_{1}+i y, x_{2}+i y, \cdots, x_{n}+i y\right)=\left(x_{1}+i y+x_{2}+i y+\cdots+x_{n}+i y\right) / n$ when all the $y$ 's are equal; otherwise,

$$
f\left(x_{1}+i y_{1}, x_{2}+i y_{2}, \cdots, x_{n}+i y_{n}\right)=\left(x_{1}+x_{2}+\cdots+x_{n}\right) / n .
$$

This example satisfies Postulates I, II, III, IV, III', $\mathrm{IV}^{\prime}, \mathrm{IV}^{\prime \prime}, \mathrm{V}, A 2, A 3$, $A 4$, but it is not the arithmetic mean. (This example would still satisfy $A 2$ and $A 3$, even if we removed the restrictions $a<1, b<1$, and $x_{i}<1$.)

Ex. $A$ X. When $n=2, f\left(x_{1}, x_{2}\right)=\left(x_{1}+x_{2}\right) / 2$; when $n>2$,

$$
f()=\left(x_{1}+x_{2}+\cdots+x_{n}\right) / n \text { or }\left|\left(x_{1}+x_{2}+\cdots+x_{n}\right) / n\right|,
$$

according as $\left(x_{1}+x_{2}+\cdots+x_{n}\right) / n$ is real or imaginary.

This example satisfies Postulates $A 1, \mathrm{I}, \mathrm{II}, \mathrm{III}, \mathrm{IV}^{\prime}, \mathrm{IV}^{\prime \prime}, \mathrm{V}$, but fails on Postulate IV.

The proofs of the theorems in the complex domain, on account of their great simplicity, are here given in full, as follows:

Theorem A (A"). Proof of A from A1, I, II, IV. (Complex domain.)

By II and $A 1, f\left(x_{1}, x_{2}, \cdots, x_{n}\right)=f\left(0, x_{1}+x_{2}, x_{3}, \cdots, x_{n}\right)$, since each side equals $f\left\{\left(x_{1}+x_{2}\right) / 2,\left(x_{1}+x_{2}\right) / 2, x_{3}, \cdots, x_{n}\right\}$. 
By successive applications of this result,

$$
f\left(x_{1}, x_{2}, \cdots, x_{n}\right)=f\left(0,0, \cdots,\left[x_{1}+x_{2}+\cdots+x_{n}\right]\right) \text {. }
$$

Now let $a=\left(x_{1}+x_{2}+\cdots+x_{n}\right) / n$, and take each $x$ equal to $a$. Then $f(a, a, \cdots, a)=f(0,0, \cdots, 0, n a)=a$, by IV.

But also $f\left(x_{1}, x_{2}, \cdots, x_{n}\right)=f(0,0, \cdots, 0, n a)$.

Hence $f\left(x_{1}, x_{2}, \cdots, x_{n}\right)=\left(x_{1}+x_{2}+\cdots+x_{n}\right) / n$.

This proof shows that Set $A 1$ determines the arithmetic mean, for all complex values of the variables.

Theorem A $\left(\mathrm{F}^{\prime \prime}\right)$. Proof of $A 1$ and IV from $A 8, A 8^{\prime}, \mathrm{I}, \mathrm{II}$. (Complex domain.)

Putting each $x=0$ in $A 8^{\prime}$, we have $f(0,0, \cdots, 0)=0$; hence putting each $x=0$ and $A=a$, in $A 8$, we have $f(a, a, \cdots, a)=a$, which is Postulate IV.

Again, putting $A=a+b, x_{1}=-a, x_{2}=-b$, and $n=2$, in $A 8$, we have $f(b, a)=a+b+f(-a,-b)$, whence, by I and $A 8^{\prime}, f(a, b)=a+b-f(a, b)$, whence $f(a, b)=(a+b) / 2$, which is Postulate $A 1$.

This proof shows that Set $A 8$ reduces to Set $A 1$, for all complex values.

Theorem A (G). Proof of $A 1$ and IV from A9, I, II. (Complex domain.)

In $A 9$, put each $x$ equal to 0 , and $A=0$; then

$$
f(0,0, \cdots, 0)=-f(0,0, \cdots, 0) \text {, }
$$

whence $f(0,0, \cdots, 0)=0$.

Hence, putting each $x$ equal to $a$, and $A=a$, we have

$$
f(0,0, \cdots, 0)=a-f(a, a, \cdots, a) \text {, }
$$

whence $f(a, a, \cdots, a)=a$, which is Postulate IV for all values of $a$.

Again, put $A=a+b, x_{1}=a, x_{2}=b$, and $n=2$, in $A 9$; then

$$
f(b, a=a)+b-f(a, b) \text {, }
$$

whence, by I, $f(a, b)=a+b-f(a, b)$, whence $f(a, b)=(a+b) / 2$, which is Postulate $A 1$ for all values of $a$.

This proof shows that Set $A 9$ reduces to Set $A 1$, for all complex values.

The sufficiency of each of the Sets $A 1, A 8, A 9$, is thus established.

In conclusion, it may be noted that of all the known sets of postulates for the arithmetic mean, the only ones that are equally available in the positive domain, the real domain, and the complex domain, are Sets $A 1$ and $A 9$. Of these, Set $A 9$ (consisting of Postulates $A 9, \mathrm{I}, \mathrm{II}$ ) would appear to be the simplest.

HARVARD UNIVERSTTY,

Caybridger, Mass. 